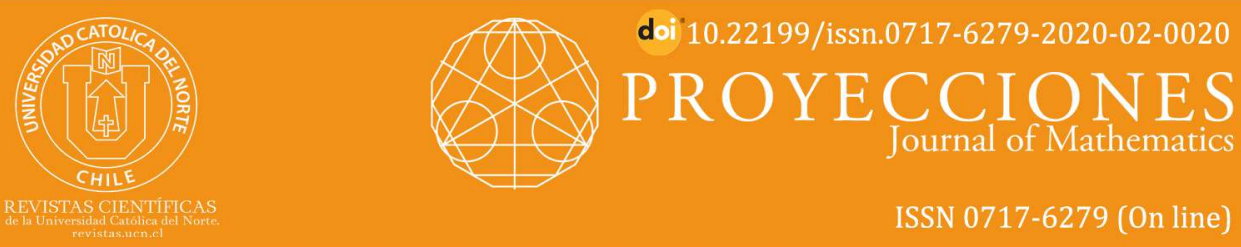

\title{
Generalized deferred Cesàro equi-statistical convergence and analogous approximation theorems
}

P. Parida ${ }^{1}$ () orcid.org/0000-0001-8239-985X

S. K. Paikray ${ }^{2}$ (1) orcid.org/0000-0003-4465-7621

B. B. Jena ${ }^{3}$ ๑) orcid.org/0000-0001-6776-0993

${ }^{1}$ Ravenshaw University, Dept. of Mathematics, Cuttack, OR, India.

- priyadarsiniparida1@gmail.com

Veer Surendra Sai University of Technology, Dept. of Mathematics, Burla, OR, India.

2曰skpaikray math@vssut.ac.in ; ${ }^{3}$ 这 bidumath.05@gmail.com

Received: January 2019 | Accepted: January 2020

\section{Abstract:}

The concept of deferred Nörlund equi-statistical convergence has recently been studied by Srivastaval et al. (see,[19]). In this paper, we have introduced the notion of equi-statistical convergence, statistical point-wise convergence and statistical uniform convergence in conjunction with the deferred statistical convergence and established a inclusion relation between them. Moreover, we have applied our idea (presumably new) of the deferred equi-statistical convergence to prove a Korovkin-type approximation theorem and demonstrated that our theorem is a non-trivial extension of some well-established Korovkintype approximation theorems which ware proved by some earlier authors. Furthermore, we have established the rate of deferred equistatistical convergence and accordingly proved a theorem. Finally, some concluding remarks and fascinating cases are shown here in support of our definitions and results.

Keywords: Statistical convergence; Cesàro deferred convergene; Deferred equi-statistical convergence; Rate of convergence; Korovkin-type approximation theorems; Banach space and positive linear operator.

MSC (2010): 40A05, 41A36, 40G15.

\section{Cite this article as (IEEE citation style)}

P. Parida, S. K. Paikray, and B. B. Jena, "Generalized deferred

Cesàro equi-statistical convergence and analogous approximation theorems", Proyecciones (Antofagasta, On line), vol. 39, no. 2, pp. 317-339, Apr. 2020, doi: 10.22199/issn.0717-6279-2020$02-0020$

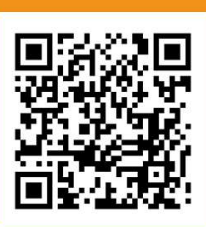

Article copyright: (C) 2020 P. Parida, S. K. Paikray, and B. B. Jena. This is an open access article distributed under the terms of the Creative Commons Licence, which permits unrestricted use and distribution provided the original author and source are credited.

(cc) BY 


\section{Introduction, Preliminaries and Motivation}

The preliminary idea of almost convergence (also known as statistical convergence) was presented by Zygmund [26] in the first edition of his monograph published in Warsaw in 1935. Next, the concept of statistical convergence was independently introduced by Fast [6] and Steinhaus [24] in the year 1950. Recently, statistical convergence has been a dynamic research area due basically to the fact that it is more general than the conventional convergence and such theory is discussed in the study of Fourier analysis, Number theory and Approximation theory. For more details, see [3], [4], [7], [8], [10], [11], [12], [14], [15], [16], [21] and [23].

Let $\mathbf{N}$ be the set of natural numbers and let $\mathcal{F} \subseteq \mathbf{N}$. Let $\mathcal{F}_{n}=\{k: k \leq$ $n$ and $k \in \mathcal{F}\}$ and suppose that $\left|\mathcal{F}_{n}\right|$ be the cardinality of $\mathcal{F}_{n}$. Then the natural density of $\mathcal{F}$ is defined by

$$
d(\mathcal{F})=\lim _{n \rightarrow \infty} \frac{\left|\mathcal{F}_{n}\right|}{n}=\lim _{n \rightarrow \infty} \frac{1}{n} \mid\{k: k \leq n \text { and } k \in \mathcal{F}\} \mid,
$$

provided the limit exists.

A given sequence $\left(x_{n}\right)$ is said to be statistically convergent to $\ell$, if for each $\epsilon>0$, the set

$$
\mathcal{F}_{\epsilon}=\left\{k: k \in \mathbf{N} \text { and }\left|x_{k}-\ell\right| \epsilon\right\}
$$

has natural density zero (see [6], [24]), that is, for each $\epsilon>0$,

$$
d\left(\mathcal{F}_{\epsilon}\right)=\lim _{n \rightarrow \infty} \frac{\left|\mathcal{F}_{\epsilon}\right|}{n} .
$$

In this case, we write

$$
\text { stat } \lim _{n \rightarrow \infty} x_{n}=\ell \text {. }
$$

It may be noted that every convergent sequence is statistically convergent, but the converse is not true.

We now recall the deferred Cesàro $D\left(a_{n}, b_{n}\right)$ summability mean as follows: 
Let $\left(a_{n}\right)$ and $\left(b_{n}\right)$ be sequences of non-negative integers such that (i) $a_{n}<b_{n}$ and (ii) $\lim _{n \rightarrow \infty} b_{n}=+\infty$, then the deferred Cesàro $D\left(a_{n}, b_{n}\right)$ mean (see [1] and [9]) is defined by

$D\left(a_{n}, b_{n}\right)=D\left(x_{n}\right)=\frac{x_{a_{n}+1}+x_{a_{n}+2}+x_{a_{n}+3}+\ldots+x_{b_{n}}}{b_{n}-a_{n}}=\frac{1}{b_{n}-a_{n}} \sum_{k=a_{n}+1}^{b_{n}} x_{k}$.

It is well known that, $D\left(a_{n}, b_{n}\right)$ is regular under conditions (i) and (ii).

Also very recently, Srivastava et al. [18] introduced the deferred weighted mean, $D_{a}^{b}(\bar{N}, p, q)$ as,

$$
t_{n}=\frac{1}{R_{a_{n}+1}^{b_{n}}} \sum_{m=a_{n}+1}^{b_{n}} p_{m} q_{m} x_{m}
$$

It will be interesting to see that, for $p_{m}=q_{m}=1, t_{n}$ is same as $D\left(x_{n}\right)$. Thus, deferred Cesàro mean is very fundamental in the study of such type of means. Here, we have considered the statistical summablity via deferred Cesàro mean in order to establish certain approximation theorems.

Let us now introduce the following definitions in support of our proposed work.

Definition 1. A sequence $\left(x_{n}\right)$ is said to be deferred statistically convergent to $\ell$ if, for every $\epsilon>0$, the set

$$
\left\{k: a_{n}<k \leq b_{n} \text { and }\left|x_{n}-\ell\right| \geq \epsilon\right\}
$$

has natural density zero, that is,

$$
\lim _{n \rightarrow \infty} \frac{1}{b_{n}-a_{n}} \mid\left\{k: a_{n}<k \leq b_{n} \text { and }\left|x_{n}-\ell\right| \geq \epsilon\right\} \mid=0 .
$$


In this case, we write

$$
\operatorname{stat}_{D C} \lim _{n \rightarrow \infty} x_{n}=\ell \text {. }
$$

Definition 2. (see [9]) A sequence $\left(x_{n}\right)$ is said to be Cesàro deferred statistically convergent to $\ell$ if, for every $\epsilon>0$, the set

$$
\left\{k: a_{n}<k \leq b_{n} \text { and }\left|D\left(x_{n}\right)-\ell\right| \geq \epsilon\right\}
$$

has natural density zero, that

is,

$$
\lim _{n \rightarrow \infty} \frac{1}{b_{n}-a_{n}} \mid\left\{k: a_{n}<k \leq b_{n} \text { and }\left|D\left(x_{n}\right)-\ell\right| \geq \epsilon\right\} \mid=0
$$

In this case, we write

$$
\text { stat }-\lim _{n \rightarrow \infty} D\left(x_{n}\right)=\ell \text { or } D C_{1} \text { (stat) }-\lim _{n \rightarrow \infty} x_{n}=\ell \text {. }
$$

Clearly, above definition can be viewed as the generalization of some existing definitions.

We now prove the following theorem which determines the inclusion relation between the deferred statistical convergence and the Cesàro deferred statistical convergence.

Theorem 1. Let a sequence $\left(x_{n}\right)$ is deferred statistically convergent to a number $\ell$, then it is Cesàro deferred statistically convergent to the same number $\ell$, but the converse is not true. 
Proof. Suppose $\left(x_{n}\right)$ is deferred statistically convergent to $\ell$. By the hypothesis, we have

$$
\lim _{n \rightarrow \infty} \frac{1}{b_{n}-a_{n}} \mid\left\{k: a_{n}<k \leq b_{n} \text { and }\left|x_{n}-\ell\right| \geq \epsilon\right\} \mid=0 .
$$

Consider two sets as follows:

$$
\mathcal{K}_{\epsilon}=\lim _{n \rightarrow \infty} \mid\left\{k: a_{n}<k \leq b_{n} \text { and }\left|x_{n}-\ell\right| \geq \epsilon\right\} \mid
$$

and

$$
\mathcal{K}_{\epsilon}^{c}=\lim _{n \rightarrow \infty} \mid\left\{k: a_{n}<k \leq b_{n} \text { and }\left|x_{n}-\ell\right|<\epsilon\right\} \mid
$$

Now,

$$
\begin{aligned}
& \left|D\left(x_{n}\right)-\ell\right|=\left|\frac{1}{b_{n}-a_{n}} \sum_{m=a_{n}+1}^{b_{n}}\left(x_{k}\right)-\ell\right| \\
& \leq\left|\frac{1}{b_{n}-a_{n}} \sum_{m=a_{n}+1}^{b_{n}}\left(x_{k}-\ell\right)\right|+|\ell|\left|\frac{1}{b_{n}-a_{n}} \sum_{m=a_{n}+1}^{b_{n}} 1-1\right| \\
& \leq \frac{1}{b_{n}-a_{n}} \sum_{m=a_{n}+1\left(k \in \mathcal{K}_{\epsilon}\right)}^{b_{n}}\left|x_{k}-\ell\right|+\frac{1}{b_{n}-a_{n}} \sum_{m=a_{n}+1\left(k \in \mathcal{K}_{\epsilon}^{c}\right)}^{b_{n}}\left|x_{k}-\ell\right|+\frac{|\ell|}{b_{n}-a_{n}} \\
& \leq \frac{1}{b_{n}-a_{n}}\left|\mathcal{K}_{\epsilon}\right|+\frac{\epsilon}{b_{n}-a_{n}}+0 \rightarrow 0 \quad \text { as } n \rightarrow \infty \quad\left(\lim _{n \rightarrow \infty} b_{n}=\infty\right),
\end{aligned}
$$

which implies that $D\left(x_{n}\right) \rightarrow \ell$. Hence, the sequence $\left(x_{n}\right)$ is Cesàro deferred statistically convergent to the same number $\ell$.

In order to prove that the converse is not true, we consider an example (below).

Example 1. Let us suppose that $a_{n}=2 n-1$ and $b_{n}=4 n-1$ and also consider the sequence $\left(x_{n}\right)$ by

$$
x_{n}=\left\{\begin{array}{cc}
0 & (n \text { is even }) \\
1 & (n \text { is odd }) .
\end{array}\right.
$$

We have,

$$
\frac{1}{b_{n}-a_{n}} \sum_{m=a_{n}+1}^{b_{n}} x_{m}=\frac{1}{2 n} \sum_{m=2 n}^{4 n-1} x_{m}=\frac{1}{2 n} n=\frac{1}{2} .
$$


Clearly, the sequence $\left(x_{n}\right)$ is neither convergent nor statistical convergent. However, it is Cesàro deferred convergent to $\frac{1}{2}$. Thus, it is Cesàro deferred statistically convergent to $\frac{1}{2}$; but the sequence $\left(x_{n}\right)$ is not deferred statistically convergent.

The concept of deferred Nörlund equi-statistical convergence was introduced by Srivastava et al. [19]. In this paper, we introduced the concept of deferred equi- statistical convergence, deferred statistical point-wise convergence and deferred statistical uniform convergence of a sequence of real valued functions and show that the deferred equi- statistical convergence lies between the deferred statistical point-wise convergence and deferred statistical uniform convergence. The inclusion relation between equi-statistical convergence and deferred equi-statistical convergence is established and it is proved that, under certain conditions, the deferred equi-statistical convergence and the equi-statistical convergence are equivalent to each other. Furthermore, we have applied our notion of deferred equi-statistical convergence to prove a Korovkin-type approximation theorem. Finally, we studied the rate of deferred equi-statistical convergence of a sequence of positive linear operators and accordingly established a theorem.

\section{Deferred Equi-statistical Convergence}

Let $\mathcal{C}(X)$ be the space of all continuous real valued functions defined on a compact subset $X$ of real numbers. $\mathcal{C}(X)$ is also a Banach space. For $f \in \mathcal{C}(X)$, we have

$$
\|f\|_{\infty}=\sup _{x \in X}\{|f(x)|\} .
$$

Let $f_{n} \in \mathcal{C}(X)$. Then, a sequence

(i) $\left(f_{n}\right)$ is said to be statistically point-wise convergent to function $f$ on $X$, if for each $\epsilon>0$ and $x \in X$, we have (see [13])

$$
\lim _{n \rightarrow \infty} \frac{R_{n}(x, \epsilon)}{n}=0,
$$

where

$$
R_{n}(x, \epsilon)=\mid\left\{k: k \leq n \text { and }\left|f_{k}(x)-f(x)\right| \epsilon\right\} \mid .
$$

Here, we write

$$
f_{n} \rightarrow f \text { (stat-pointwise) on } X \text {. }
$$


(ii) $\left(f_{n}\right)$ is said to be equi-statistically convergent to function $f$ on $X$, if for each $\epsilon>0$, we have (see [13])

$$
\lim _{n \rightarrow \infty} \frac{R_{n}(x, \epsilon)}{n}=0
$$

uniformly with respect to $x \in X$, which means that

$$
\lim _{n \rightarrow \infty} \frac{\left\|R_{n}(x, \epsilon)\right\|_{C(X)}}{n}=0
$$

for every $\epsilon>0$, where

$$
R_{n}(x, \epsilon)=\left|\left\{k \leq n:\left|f_{k}(x)-f(x)\right| \geq \epsilon\right\}\right| .
$$

Here, we write

$$
f_{n} \rightarrow f \text { (equi-stat) on } X
$$

(iii) $\left(f_{n}\right)$ is said to be statistically uniform convergent to function $f$ on $X$, if for each $\epsilon>0$ and for each $x \in X$, we have (see [13])

$$
\lim _{n \rightarrow \infty} \frac{R_{n}(x, \epsilon)}{n}=0
$$

where

$$
R_{n}(x, \epsilon)=\left|\left\{k: k \leq n:\left\|f_{k}(x)-f(x)\right\|_{C(X)} \epsilon\right\}\right| .
$$

Here, we write

$$
f_{n} f \text { (stat-uniform) on } X \text {. }
$$

We now introduce the following definitions for our proposed study.

Definition 3. A sequence of functions $\left(f_{n}\right)$ is said to be deferred statistically point-wise convergent to a function $f$ on $X$, if for each $\epsilon>0$ and for every $x \in X$, we have

$$
\lim _{n \rightarrow \infty} \frac{L_{n}(x, \epsilon)}{b_{n}-a_{n}}=0
$$


where

$$
L_{n}(x, \epsilon)=\mid\left\{m: a_{n} \leq m \leq b_{n} \text { and }\left|f_{m}(x)-f(x)\right| \epsilon\right\} \mid .
$$

Here, we denote the limit by,

$$
f_{n} \rightarrow f\left(D\left(x_{n}\right) \text {-stat-pointwise }\right) .
$$

Definition 4. A sequence of functions $\left(f_{n}\right)$ is said to be deferred equistatistically convergent to a function $f$ defined on $X$, if for each $\epsilon>0$,

$$
\lim _{n \rightarrow \infty} \frac{L_{n}(x, \epsilon)}{a_{n}-b_{n}}=0,
$$

uniformly with respect to $x \in X$, that is,

$$
\lim _{n \rightarrow \infty} \frac{\left\|L_{n}(x, \epsilon)\right\|_{C(X)}}{a_{n}-b_{n}}=0
$$

where

$$
L_{n}(x, \epsilon)=\frac{1}{a_{n}-b_{n}} \mid\left\{m: a_{n} \leq m \leq b_{n} \text { and }\left|f_{m}(x)-f(x)\right| \epsilon\right\} \mid .
$$

Here, we denote the limit by,

$$
f_{n} \rightarrow f\left(D\left(x_{n}\right) \text {-equi-stat }\right) .
$$

Definition 5. A sequence of functions $\left(f_{n}\right)$ is said to be deferred statistically uniform convergent to a function $f$ on $X$, if for each $\epsilon>0$, we have

$$
\lim _{n \rightarrow \infty} \frac{L_{n}(x, \epsilon)}{a_{n}-b_{n}}=0,
$$

where

$$
L_{n}(x, \epsilon)=\mid\left\{m: a_{n} \leq m \leq b_{n} \text { and }\left\|f_{m}(x)-f(x)\right\|_{C(X)} \epsilon\right\} \mid .
$$

In this case, we write

$$
f_{n} f\left(D\left(x_{n}\right) \text {-stat-uniform }\right) .
$$


From the above definitions, we have a trivial result in the form of the following lemma.

Lemma 1. The following implications are true

$$
\begin{gathered}
f_{n} f\left(D\left(x_{n}\right) \text {-stat-uniform }\right) \Longrightarrow f_{n} \rightarrow f\left(D\left(x_{n}\right) \text {-equi-stat }\right) \\
\Longrightarrow f_{n} \rightarrow f\left(D\left(x_{n}\right) \text {-stat-pointwise }\right)
\end{gathered}
$$

Moreover, it may be noted that, the above inclusions are strict, that is, the reverse implications of Lemma 1 are not always true. For this propose we present the following examples.

Example 2. In this example we illustrate that, the first inclusion is strict.

Let

$$
a_{n}=n, \quad b_{n}=2 n \text { and } g_{n}: X \rightarrow[0,1] \quad(n \in \mathbf{N})
$$

be sequence of continuous real valued functions, defined by

$$
g_{n}(x)= \begin{cases}2^{n+1}\left(x-\frac{1}{2^{n}}\right), & x \in\left[\frac{1}{2^{n}}, \frac{1}{2^{n-1}}-\frac{1}{2^{n+1}}\right] \\ -2^{n+1}\left(x-\frac{1}{2^{n-1}}\right), & x \in\left[\frac{1}{2^{n-1}}-\frac{1}{2^{n+1}}, \frac{1}{2^{n-1}}\right] \\ 0, & \text { otherwise }\end{cases}
$$

then, for every $\epsilon>0$, we have

$\frac{1}{a_{n}-b_{n}} \mid\left\{m: a_{n} \leq m \leq b_{n}\right.$ and $\left.\left|g_{m}(x)-g(x)\right| \epsilon\right\} \mid \leq \frac{1}{a_{n}-b_{n}} \rightarrow 0$, as $n \rightarrow \infty$ 
uniformly on $X$. This implies

$$
g_{n} \rightarrow 0\left(D\left(x_{n}\right) \text {-equi-stat }\right)
$$

But since,

$$
\sup _{x \in[0,1]}\left|g_{n}(x)\right|=1(n \in \mathbf{N})
$$

so

$$
g_{n} 0\left(D\left(x_{n}\right) \text {-uniform-stat }\right)
$$

does not hold true.

Example 3. In this example we illustrate that, the second inclusion is strict.

Let

$$
a_{n}=n, b_{n}=2 n \text { and } g_{n}: X=[0,1] \quad(n \in \mathbf{N})
$$

be sequence of continuous real valued functions, defined by

$$
g_{n}(x)=x^{n}, n \in \mathbf{N}
$$

Suppose that,

$$
\lim _{n \rightarrow \infty} g_{n}(x)=g(x), \text { for } x \in[0,1]
$$

then

$$
g_{n}(x) \rightarrow g(x)\left(D\left(x_{n}\right) \text {-pointwise-stat }\right)
$$

If we take $\epsilon=\frac{1}{7}$, then for all $n \in \mathbf{N}$, there exists $r>\mathbf{N}$ such that $r \in\left[a_{n}+1, b_{n}\right]$ and for every $x \in\left(\sqrt[n]{\frac{1}{7}}, 1\right)$, we have

$$
\left|g_{r}(x)=\right| x^{r}|\geq|\left(\sqrt[n]{\frac{1}{7}}\right)^{r}|\geq|\left(\sqrt[r]{\frac{1}{7}}\right)^{r} \mid=\frac{1}{7}
$$

Thus, the condition $g_{n} \rightarrow 0$ ( $D\left(x_{n}\right)$-equi-stat) does not hold true. 


\section{A Korovkin-type Theorem}

A few mathematicians have worked on extending or generalizing the Korovkin theorems in many ways based on several aspects, including function spaces, abstract Banach lattices, Banach algebras, Banach spaces and so on. This theory is extremely valuable in real analysis, functional analysis, harmonic analysis, measure theory, probability theory, summability theory and partial differential equations, etc. But the premier applications are concerned with constructive approximation theory which utilizes it as a significant tool. Indeed, even today, the improvement of Korovkin-type approximation theory is a long way from finished. For more results related to the Korovkin-type theorems (see [5], [9], [17], [20] and [22]).

Let $\mathcal{C}[a, b]$ be the linear space of all real-valued continuous functions $f$ defined on $[a, b]$ and suppose that $\mathcal{L}: \mathcal{C}[a, b] \rightarrow \mathcal{C}[a, b]$ be a linear operator. We say that $\mathcal{L}$ is positive if,

$$
\mathcal{L}(f ; x) 0 \text {, wheneever } x \in[a, b] .
$$

Also, it is known that $\mathcal{C}[a, b]$ is a Banach space. We have for $f \in \mathcal{C}[a, b]$, the norm of $f$ denoted by $\|f\|$ is given by,

$$
\|f\|_{\infty}=\sup _{x \in[a, b]}|f(x)| .
$$

The classical Korovkin approximation theorem states as follows [14]:

Let $\mathcal{L}_{n}: \mathcal{C}[a, b] \rightarrow \mathcal{C}[a, b]$ be a sequence of positive linear operators and $f \in \mathcal{C}[a, b]$. Then

$\lim _{n \rightarrow \infty}\left\|\mathcal{L}_{n}(f ; x)-f(x)\right\|_{\infty}=0$, if and only if $\lim _{n \rightarrow \infty}\left\|\mathcal{L}_{n}\left(f_{i} ; x\right)-f_{i}(x)\right\|_{\infty}=0,(i=0,1,2)$,

where

$$
f_{0}(x)=1, f_{1}(x)=x \text { and } f_{2}(x)=x^{2}
$$

In this section, we extend the result of Karakus et al. [13] via deferred equi-statistical convergence and established the following theorem. 
Theorem 2. Let $\mathcal{L}_{m}$ be a sequence of positive linear operators from $\mathcal{C}[a, b]$ into itself and let $f \in \mathcal{C}[X]$. Then,

$$
\mathcal{L}_{m}(f ; x) \rightarrow f(x),\left(D\left(x_{n}\right) \text {-equi-stat }\right) \text { on } X
$$

if and only if

$$
\mathcal{L}_{m}\left(f_{i}, x\right) \rightarrow f_{i}(x),\left(D\left(x_{n}\right) \text {-equi-stat }\right) \text { on } X,\left(f_{i}(x)=x^{i} ; i=\{0,1,2\}\right) .
$$

Proof. Since each $f_{i}(x)=x^{i} \in \mathcal{C}(X), i=\{0,1,2\}$ are continuous, the implication

$$
(3.1) \Longrightarrow(3.2)
$$

is fairly obvious. In order to complete the proof of Theorem 2, we first assume that (3.2) holds true. Let $f \in \mathcal{C}[X]$, then there exist a constant $\mathcal{K}>0$ such that

$$
|f(x)| \leq \mathcal{K} \text {, for all } x \in X \text {. }
$$

This implies

$$
|f(y)-f(x)| \leq 2 \mathcal{K} \quad(x, y \in X) .
$$

Clearly, for given $\epsilon>0$, there exist $\delta>0$ such that

$$
|f(y)-f(x)|<\epsilon
$$

whenever

$$
|y-x|<\delta, \text { for all } x, y \in X
$$

Let us choose $\varphi=\varphi(y, x)=(y-x)^{2}$. If $|y-x| \delta$, then we obtain 


$$
|f(y)-f(x)|<\frac{2 \mathcal{K}}{\delta^{2}} \varphi(y, x)
$$

From equation (3.4) and (3.5), we get

$$
|f(y)-f(x)|<\epsilon+\frac{2 \mathcal{K}}{\delta^{2}} \varphi(y, x) .
$$

This implies that,

$$
-\epsilon-\frac{2 \mathcal{K}}{\delta^{2}} \varphi(y, x)(y)-f(x) \leq \epsilon+\frac{2 \mathcal{K}}{\delta^{2}} \varphi(y, x) .
$$

Now by applying the operator $\mathcal{L}_{m}(1, x)$ to this inequality $\left(\mathcal{L}_{m}(1, x)\right.$ is monotone and linear), we have

$$
\mathcal{L}_{m}(1, x)\left(-\epsilon-\frac{2 \mathcal{K}}{\delta^{2}} \varphi(y, x)\right) \leq \mathcal{L}_{n}(1, x)(f(y)-f(x)) \leq \mathcal{L}_{m}(1, x)\left(\epsilon+\frac{2 \mathcal{K}}{\delta^{2}} \varphi(y, x)\right)
$$

Note that $x$ is fixed and so $f(x)$ is a constant number.

Therefore,

$$
\begin{aligned}
&-\epsilon \mathcal{L}_{m}(1, x)-\frac{2 \mathcal{K}}{\delta^{2}} \mathcal{L}_{m}(\varphi, x) \leq \mathcal{L}_{m}(f, x)-f(x) \mathcal{L}_{m}(1, x) \\
& \leq \epsilon \mathcal{L}_{m}(1, x)+\frac{2 \mathcal{K}}{\delta^{2}} \mathcal{L}_{m}(\varphi, x)
\end{aligned}
$$

But

$$
\mathcal{L}_{m}(f, x)-f(x)=\left[\mathcal{L}_{m}(f, x)-f(x) \mathcal{L}_{m}(1, x)\right]+f(x)\left[\mathcal{L}_{m}(1, x)-1\right]
$$

Using (3.8) and (3.9), we have

(3.10) $\mathcal{L}_{m}(f, x)-f(x)<\epsilon \mathcal{L}_{m}(1, x)+\frac{2 \mathcal{K}}{\delta^{2}} \mathcal{L}_{m}(\varphi, x)+f(x)\left[\mathcal{L}_{m}(1, x)-1\right]$. 
Next, estimate $\mathcal{L}_{m}(\varphi, x)$ as,

$$
\begin{aligned}
\mathcal{L}_{m}(\varphi, x) & =\mathcal{L}_{m}\left((y-x)^{2}, x\right)=\mathcal{L}_{m}\left(y^{2}-2 x y+x^{2}, x\right) \\
& =\mathcal{L}_{m}\left(y^{2}, x\right)-2 x \mathcal{L}_{m}(y, x)+x^{2} \mathcal{L}_{m}(1, x) \\
& =\left[\mathcal{L}_{m}\left(y^{2}, x\right)-x^{2}\right]-2 x\left[\mathcal{L}_{m}(y, x)-x\right]+x^{2}\left[\mathcal{L}_{m}(1, x)-1\right]
\end{aligned}
$$

Using (3.10), we obtain

$$
\begin{aligned}
& \mathcal{L}_{m}(f, x)-f(x)<\epsilon \mathcal{L}_{m}(1, x)+\frac{2 K}{\delta^{2}}\left\{\left[\mathcal{L}_{m}\left(y^{2}, x\right)-x^{2}\right]-2 x\left[\mathcal{L}_{m}(y, x)-x\right]+x^{2}\left[\mathcal{L}_{m}(1, x)-1\right]\right\} \\
&+f(x)\left[\mathcal{L}_{m}(1, x)-1\right] \\
&=\epsilon\left[\mathcal{L}_{m}(1, x)-1\right]+\epsilon+\frac{2 \mathcal{K}}{\delta^{2}}\left\{\left[\mathcal{L}_{m}\left(y^{2}, x\right)-x^{2}\right]-2 x\left[\mathcal{L}_{m}(y, x)-x\right]\right. \\
&\left.+x^{2}\left[\mathcal{L}_{m}(1, x)-1\right]\right\}+f(x)\left[\mathcal{L}_{m}(1, x)-1\right] .
\end{aligned}
$$

Since $\epsilon$ is arbitrary, we can write

$$
\begin{aligned}
&\left|\mathcal{L}_{m}(f, x)-f(x)\right| \leq(\epsilon\left.+\frac{2 \mathcal{K}}{\delta^{2}}+\mathcal{K}\right)\left|\mathcal{L}_{m}(1, x)-1\right| \\
& \quad+\frac{4 \mathcal{K}}{\delta^{2}}\left|\mathcal{L}_{m}(y, x)-x\right|+\frac{2 \mathcal{K}}{\delta^{2}}\left|\mathcal{L}_{m}\left(y^{2}, x\right)-x^{2}\right| \\
&(3.11) \quad \leq \mathcal{B}\left(\left|\mathcal{L}_{m}(1, x)-1\right|+\left|\mathcal{L}_{m}(y, x)-x\right|+\left|\mathcal{L}_{m}\left(y^{2}, x\right)-x^{2}\right|\right),
\end{aligned}
$$

where

$$
\mathcal{B}=\max \left(\epsilon+\frac{2 \mathcal{K}}{\delta^{2}}+\mathcal{K}, \frac{4 \mathcal{K}}{\delta^{2}}, \frac{2 \mathcal{K}}{\delta^{2}}\right) .
$$

Now replacing

$$
\mathcal{L}_{m}(f ; x) \text { by } \frac{1}{b_{n}-a_{n}} \sum_{m=a_{n}+1}^{b_{n}} T_{m}(f ; x)
$$


and then by $L_{m}(f ; x)$ in $(3.11)$, we have for a given $r>0$, there exists $\epsilon>0$, such that $\epsilon<r$. Then, by setting

$$
L_{m}(x ; r)=\left\{m: a_{n}<m \leq b_{n} \text { and }\left|\frac{1}{b_{n}-a_{n}} \sum_{m=a_{n}+1}^{b_{n}} T_{m}(f ; x)-f(x)\right| r\right\}
$$

and for $i=0,1,2$,

$L_{i, m}(x ; r)=\left\{m: a_{n}<m \leq b_{n}\right.$ and $\left.\left|\frac{1}{b_{n}-a_{n}} \sum_{m=a_{n}+1}^{b_{n}} T_{m}\left(f_{i} ; x\right)-f_{i}(x)\right| \frac{r-\epsilon}{3 \mathcal{B}}\right\}$,

we obtain,

$$
L_{m}(x ; r) \leq \sum_{i=0}^{2} L_{i, m}(x ; r)
$$

Clearly,

$$
\frac{\left\|L_{m}(x ; r)\right\|_{\mathcal{C}(X)}}{b_{n}-a_{n}} \leq \sum_{i=0}^{2} \frac{\left\|L_{i, m}(x ; r)\right\|_{\mathcal{C}(X)}}{b_{n}-a_{n}}
$$

Now, using the above assumption about the implications in (3.2) and by Definition 4, the right-hand side of (3.12) is seen to tend to zero as $n \rightarrow \infty$. Consequently, we get

$$
\lim _{n \rightarrow \infty} \frac{\left\|L_{m}(x ; r)\right\|_{\mathcal{C}(X)}}{b_{n}-a_{n}}=0(r>0) .
$$

Therefore, the implication (3.1) holds true.

This completes the proof of Theorem 2 .

Example 4. Let $X=[0,1]$ and we consider the Bernstine polynomial $B_{n}(f ; x)$ on $\mathcal{C}[0,1]$.

Let $\mathcal{L}_{m}: \mathcal{C}[0,1] \rightarrow \mathcal{C}[0,1]$ be sequence of operators defined as follows

$$
\mathcal{L}_{n}(f ; x)=\left[1+g_{n}(x)\right] x(1+x D) B_{n}(f ; x), f \in \mathcal{C}[0,1]
$$


where the operator

$$
x(1+x D)\left(D=\frac{d}{d x}\right),
$$

which was earlier used by Al-Salam [2] (For more about the operator see Viskov and Srivastava [25]) and suppose that $f_{n}(x)$ be a sequence as considered in our Example 2. Now, we have

$$
\begin{gathered}
\mathcal{L}_{n}\left(f_{0} ; x\right)=\left[1+g_{n}(x)\right] x(1+x D) f_{0}(x)=\left[1+g_{n}(x)\right] x, \\
\mathcal{L}_{n}\left(f_{1} ; x\right)=\left[1+g_{n}(x)\right] x(1+x D) f_{1}(x)=\left[1+g_{n}(x)\right] x(1+x), \\
\mathcal{L}_{n}\left(f_{2} ; x\right)=\left[1+g_{n}(x)\right] x(1+x D)\left\{f_{2}(x)\left(\frac{n+2}{n+1}\right)+\frac{x}{n+1}\right\} \\
=\left[1+g_{n}(x)\right]\left\{x^{2}\left[\left(\frac{n+2}{n+1}\right) x+2\left(\frac{1}{n+1}\right)+2 x\left(\frac{n+2}{n+1}\right)\right]\right\} .
\end{gathered}
$$

Next, as

$$
f_{n} \rightarrow f=0\left(D\left(x_{n}\right) \text {-equi-stst }\right) \text { on }[0,1]
$$

with $f_{n}(x)$ as defined in Example 2, so we conclude that

$$
\mathcal{L}_{n}\left(f_{i} ; x\right) \rightarrow f_{i}\left(D\left(x_{n}\right) \text {-equi-stst }\right) \text { on }[0,1] \text { for each } i=0,1,2 .
$$

Thus, by Theorem 2, we see that

$$
L_{n}(f ; x) \rightarrow f\left(D\left(x_{n}\right) \text {-equi-stst }\right) \text { on }[0,1]
$$

for all $f \in C[0,1]$.

Moreover, since $\left(f_{n}\right)$ is not $D\left(x_{n}\right)$-statistically uniform convergent to the function $f=0$ on the interval $[0,1]$, we can say that the result of Karakuş et al. [13] (p. 1067, Theorem 2.1) does not hold for our operators defined by (3.13). Furthermore, since $\left(f_{n}\right)$ is not uniformly convergent (in the usual sense) to the function $f=0$ on $[0,1]$, the usual Korovkin-type theorem also does not work here (see [14]). However, our Theorem 2 fairly holds true under the operators defined by (3.13). 


\section{Rate of Deferred Cesàro Eqiu-statistical Convergence}

In this section, we will study the rate of deferred equi-statistical convergence of a sequence of positive linear operators defined on $\mathcal{C}(X)$ with the help of the modulus of continuity.

We now present below a definition.

Definition 6. Let $\left(s_{n}\right)$ be a positive non-increasing sequence. A sequence $\left(f_{n}\right)$ is deferred equi-statistically convergent to a function $(f)$ with rate of $o\left(s_{n}\right)$, if for every $\epsilon>0$,

$$
\lim _{n \rightarrow \infty} \frac{\Omega_{n}(x, \epsilon)}{s_{n}\left(b_{n}-a_{n}\right)}=0
$$

uniformly

with respect to $x \in X$, or equivalently if

$$
\lim _{n \rightarrow \infty} \frac{\left\|\Omega_{n}(x, \epsilon)\right\|_{C(X)}}{s_{n}\left(b_{n}-a_{n}\right)}=0,
$$

where

$$
\Omega_{n}(x, \epsilon)=\mid\left\{m: a_{n} \leq m \leq b_{n} \text { and }\left|f_{m}(x)-f(x)\right| \epsilon\right\} \mid .
$$

In this case, we may write

$$
f_{n}(x)-f(x)=o\left(s_{n}\right)\left(D\left(x_{n}\right) \text {-equi-stat }\right) \text { on } X .
$$

We now prove a lemma as follows.

Lemma 2. Let $\left(f_{n}\right)$ and $\left(g_{n}\right)$ belonging to $\mathcal{C}[X]$ satisfying

$$
f_{n}(x)-f(x)=o\left(s_{n}\right)\left(D\left(x_{n}\right) \text {-equi-stat }\right) \text { on } X
$$

and

$$
g_{n}(x)-g(x)=o\left(c_{n}\right)\left(D\left(x_{n}\right) \text {-equi-stat }\right) \text { on } X .
$$

Then the following conditions fairly hold true 
(i) $\left(f_{n}(x)+g_{n}(x)\right)-(f(x)+g(x))=o\left(d_{n}\right)\left(D\left(x_{n}\right)\right.$-equi-stat $)$ on $X$,

(ii) $\left(f_{n}(x)-f(x)\right)\left(g_{n}(x)-g(x)\right)=o\left(s_{n} c_{n}\right)\left(D\left(x_{n}\right)\right.$-equi-stat $)$ on $X$,

(iii) $\lambda\left(f_{n}(x)-f(x)\right)=o\left(s_{n}\right)\left(D\left(x_{n}\right)\right.$-equi-stat) on $X$ for any scalar $\lambda$,

(iv) $\sqrt{\left|f_{n}(x)-f(x)\right|}=o\left(s_{n}\right)\left(D\left(x_{n}\right)\right.$-equi-stat) on $X$,

where

$$
d_{n}=\max \left\{s_{n}, c_{n}\right\}
$$

Proof. In order to prove the condition (i), for $\epsilon>0$ and $x \in X$, we define the following sets:

$$
\begin{aligned}
G_{n}(x, \epsilon) & =\mid\left\{m: a_{n} \leq m \leq b_{n} \text { and }\left|\left(f_{m}+g_{m}\right)(x)-(f+g)(x)\right| \epsilon\right\} \mid, \\
G_{0, n}(x, \epsilon) & =\mid\left\{m: a_{n} \leq m \leq b_{n} \text { and }\left|f_{m}(x)-f(x)\right| \frac{\epsilon}{2}\right\} \mid,
\end{aligned}
$$

and

$$
G_{1, n}(x, \epsilon)=\mid\left\{m: a_{n} \leq m \leq b_{n} \text { and }\left|g_{m}(x)-g(x)\right| \frac{\epsilon}{2}\right\} \mid .
$$

Clearly, we have

$$
G_{n}(x, \epsilon) \subseteq G_{0, n}(x, \epsilon) \cup G_{1, n}(x, \epsilon) .
$$

Moreover, since

$$
d_{n}=\max \left\{s_{n}, c_{n}\right\},
$$

by the condition (3.1) of Theorem 2, we obtain

$$
\frac{\left\|G_{n}(x, \epsilon)\right\|_{\mathcal{C}(X)}}{s_{n}\left(b_{n}-a_{n}\right)} \leq \frac{\left\|G_{0, n}(x, \epsilon)\right\|_{\mathcal{C}(X)}}{s_{n}\left(b_{n}-a_{n}\right)}+\frac{\left\|G_{1, n}(x, \epsilon)\right\|_{\mathcal{C}(X)}}{s_{n}\left(b_{n}-a_{n}\right)}
$$

Again, by taking condition (3.2) of the Theorem 2, we obtain 


$$
\frac{\left\|G_{n}(x, \epsilon)\right\|_{\mathcal{C}(X)}}{s_{n}\left(b_{n}-a_{n}\right)}=0,
$$

which completes the proof of condition (i). Next, the conditions (ii)-(iv) being similar to (i), so by similar lines it can be proved.

Which completes the proof of the lemma.

Furthermore, we recall that the modulus of continuity of a function $f \in \mathcal{C}[X]$ is defined by

$$
\omega(f, \delta)=\sup _{|y-x| \leq \delta x, y \in X}|f(y)-f(x)|(\delta>0) .
$$

Then we state and prove the following result.

Theorem 3. Let $X \subset \mathbf{R}$ be a compact set, and let $\mathcal{L}_{m}: \mathcal{C}(X) \rightarrow \mathcal{C}(X)$ be a sequence of positive linear operators. Suppose that the following conditions hold true,

(i) $\mathcal{L}_{m}(1, x)-1=o\left(s_{n}\right)\left(D\left(x_{n}\right)\right.$-equi-stat $)$ on $X$,

(ii) $\omega\left(f, \delta_{n}\right)=o\left(c_{n}\right)\left(D\left(x_{n}\right)\right.$-equi-stat $)$ on $X$,

where

$$
\delta_{n}(x)=\sqrt{\mathcal{L}_{m}\left(\varphi^{2} ; x\right)} \text { and } \varphi(y)=y-x .
$$

Then, for all $f \in \mathcal{C}(X)$, the following statement holds:

$$
\mathcal{L}_{m}(f, x)-f=o\left(d_{n}\right) \quad\left(D\left(x_{n}\right) \text {-equi-stat }\right) \text { on } X,
$$

where

$$
d_{n}=\max \left\{s_{n}, c_{n}\right\}
$$


Proof. Let $f \in \mathcal{C}[X]$ and $x \in X$. Then it is well known that, $\left|\mathcal{L}_{m}(f ; x)-f(x)\right| \leq \mathcal{L}_{m}(f(y)-f(x) ; x)+|f(x)|\left|\mathcal{L}_{m}(1 ; x)-1\right|$

$$
\begin{aligned}
& \leq \mathcal{L}_{m}\left(\frac{x-y}{\delta}+1 ; x\right) \omega(f, \delta)+|f(x)|\left|\mathcal{L}_{m}(1 ; x)-1\right| \quad \text { (by equation 4.3) } \\
& \leq\left(\mathcal{L}_{m}(1 ; x)+\frac{1}{\delta^{2}} \mathcal{L}_{m}\left(\varphi^{2} ; x\right)\right) \omega(f, \delta)+|f(x)|\left|\mathcal{L}_{m}(1 ; x)-1\right| \quad\left(\varphi=(x-y)^{2}\right) \\
& \leq \omega(f, \delta)\left|\mathcal{L}_{m}(1 ; x)-1\right|+\omega(f, \delta)+|f(x)|\left|\mathcal{L}_{m}(1 ; x)-1\right|+\omega(f, \delta) \frac{1}{\delta^{2}} \mathcal{L}_{m}\left(\varphi^{2} ; x\right),
\end{aligned}
$$

and if we choose $\delta=\delta_{n}=\sqrt{\mathcal{L}_{m}\left(\varphi^{2} ; x\right)}$, we have

$\left\|\mathcal{L}_{m}(f ; x)-f(x)\right\|_{\infty} \leq \omega(f, \delta)\left\|\mathcal{L}_{m}(1 ; x)-1\right\|_{\infty}+\|f(x)\|_{\infty}\left\|\mathcal{L}_{m}(1 ; x)-1\right\|_{\infty}+2 \omega(f, \delta)$ $\leq M\left\{\omega(f, \delta)\left\|\mathcal{L}_{m}(1 ; x)-1\right\|_{\infty}+\left\|\mathcal{L}_{m}(1 ; x)-1\right\|_{\infty}+\omega(f, \delta)\right\}$,

where

$$
M=\left\{\|f\|_{\infty}, 2\right\} .
$$

Now, in the above inequality by using the conditions (i) and (ii) of Theorem 3 , in conjunction with Lemma 2, we arrive at the consequence (4.4) of Theorem 3 .

This completes the proof of the theorem.

\section{Concluding Remark and Observations}

In this concluding section of our investigation, we present several further remarks and observations concerning to various results which we have proved here.

Remark 2. Let $\left(f_{n}\right)_{n \in \mathbf{N}}$ be the sequence of functions given in Example 2, then as

$$
f_{n} \rightarrow 0\left(D\left(x_{n}\right) \text {-equi-stat }\right) \text { on }[0,1]
$$

so

$$
\mathcal{L}_{m}\left(f_{i}, x\right) \rightarrow f_{i}\left(D\left(x_{n}\right) \text {-equi-stat }\right) \text { on }[0,1] ; i=0,1,2 .
$$

Therefore, by Theorem 2, we write

$$
\mathcal{L}_{m}(f, x) \rightarrow f\left(D\left(x_{n}\right) \text {-equi-stat }\right) \text { on }[0,1] \text { and for all } f \in X .
$$

However, since $\left(f_{n}\right)$ is not deferred Cesàro statistically uniform convergent to the function $f=0$ on the interval $[0,1]$, and $\left(f_{n}\right)$ is not uniformly 
convergent (in the ordinary sense) to the function $f=0$ on $[0,1]$, the classical Korovkin-type theorem does not work for the operators defined by (3.13). Therefore, this application clearly shows that our Theorem 2 is a non-trivial generalization of the classical and statistical version of Korovkintype theorem (see [13], [14]).

Remark 3. Let us suppose we replace the condition (i) and (ii) in our Theorem 3 by the following condition

$$
\mathcal{L}_{m}\left(f_{i}, x\right)-f_{i}=o\left(s_{n_{i}}\right)\left(D\left(x_{n}\right) \text {-equi-stat }\right) \text { on } X ; i=0,1,2 \text {. }
$$

Then, since

$$
\mathcal{L}_{m}\left(\varphi^{2} ; x\right)=\mathcal{L}_{m}\left(y^{2} ; x\right)-2 x \mathcal{L}_{m}(y ; x)+x^{2} \mathcal{L}_{m}(1 ; x),
$$

so we can write

$$
\mathcal{L}_{m}\left(\varphi^{2} ; x\right) \leq K \sum_{i=0}^{2}\left|\mathcal{L}_{m}\left(f_{i} ; x\right)-f_{i}(x)\right|
$$

where

$$
K=1+2\left\|f_{1}\right\|_{\mathcal{C}[X]}+\left\|f_{2}\right\|_{\mathcal{C}[X]} .
$$

Now it follows from (5.3), (5.4) and Lemma 2 that

$$
\delta_{n}=\sqrt{\mathcal{L}_{m}\left(\varphi^{2}\right)}=o\left(u_{n}\right)\left(D\left(x_{n}\right) \text {-equi-stat }\right) \text { on } X,
$$

where

$$
o\left(u_{n}\right)=\max \left\{s_{n_{0}}, s_{n_{1}}, s_{n_{2}}\right\}
$$

Hence,

$$
\omega(f, \delta)=o\left(u_{n}\right)\left(D\left(x_{n}\right) \text {-equi-stat }\right) \text { on } X .
$$

Using (5.5) in Theorem 3, we immediately see that, for all $f \in \mathcal{C}[X]$,

$$
\mathcal{L}_{m}(f ; x)-f(x)=o\left(u_{n}\right)\left(D\left(x_{n}\right) \text {-equi-stat }\right) \text { on } X .
$$

Therefore, if we use the condition (5.3) in Theorem 3 instead of (i) and (ii), then we obtain the rates of deferred Cesàro equi-statistical convergence of the sequence of positive linear operators in Theorem 3. 


\section{References}

[1] R. P. Agnew, "On deferred cesaro means", The annals of mathematics, vol. 33, no. 3, pp. 413-421, Jul. 1932, doi: 10.2307/1968524.

[2] W. A. Al-Salam, "Operational representations for the Laguerre and other polynomials", Duke mathematical journal, vol. 31, no. 1, pp. 127-142, 1964, doi: 10.1215/S0012-7094-64-03113-8.

[3] N. L. Braha, V. Loku, and H. Srivastava, " $\Lambda 2$-Weighted statistical convergence and Korovkin and Voronovskaya type theorems", Applied mathematics and computation, vol. 266, pp. 675-686, Sep. 2015, doi: 10.1016/j.amc.2015.05.108.

[4] N. L. Braha, H. M. Srivastava, and S. A. Mohiuddine, "A Korovkin's type approximation theorem for periodic functions via the statistical summability of the generalized de la Vallée Poussin mean", Applied mathematics and computation, vol. 228, pp. 162-169, Feb. 2014, doi: 10.1016/j.amc.2013.11.095.

[5] A. A. Das, B. B. Jena, S. K. Paikray and R. K. Jati, "Statistical deferred weighted summability and associated Korovokin-type approximation theorem", Nonlinear science letters a mathematics, physics and mechanics, vol. 9, no. 3, pp. 238245, 2018.

[6] H. Fast, "Sur la convergence statistique", Colloquium mathematicum, vol. 2, no. 3-4, pp. 241-244, 1951. [On line]. Avalaible: https://bit.ly/2wZt45c

[7] B. B. Jena, S. K. Paikray, "Product of statistical probability convergence and its applications to Korovkin-type theorem", Miskolc mathematical notes, vol. 20, no. 2, pp. 969-984, 2019. [On line]. Avalaible: https://bit.ly/3eFkqKa

[8] B. Jena, S. Paikray and U. Misra, "Inclusion theorems on general convergence and statistical convergence of $(\mathrm{L}, 1,1)$-summability using generalized Tauberian conditions", Tamsui Oxford journal of information and mathematical sciences, vol. 31, pp. 101-115, 2017.

[9] B. Jena, S. Paikray, and U. Misra, "Statistical deferred Cesàro summability and its applications to approximation theorems", Filomat, vol. 32, no. 6, pp. 23072319, 2018, doi: 10.2298/FIL1806307J.

[10] B. B. Jena, S. K. Paikray, and U. K. Misra, "Approximation of periodic functions via statistical $\beta$-summability and its applications to approximation theorems", Indian journal of industrial and applied mathematics, vol. 10, no. 1si, pp. 71-86, 2019, doi: 10.5958/1945-919X.2019.00006.9.

[11] B. B. Jena, S. K. Paikray, S. A. Mohiuddine, and V. N. Mishra, "Relatively equistatistical convergence via deferred Nörlund mean based on difference operator of fractional-order and related approximation theorems", AIMS mathematics, vol. 5, no. 1, pp. 650-672, 2020, doi: 10.3934/math.2020044.

[12] B. B. Jena, S. K. Paikray, P. Parida and H. Dutta, "Results on Tauberian theorem for Cesàro summable double sequences of fuzzy numbers", Kragujevac journal of mathematics, vol. 44, no. 4, pp. 495-508, 2020. [On line]. Avalaible: https://bit.ly/3axvHJs

[13] S. Karakuş, K. Demirci, and O. Duman, "Equi-statistical convergence of positive linear operators", Journal of mathematical analysis and applications, vol. 339, no. 2, pp. 1065-1072, Mar. 2008, doi: 10.1016/j.jmaa.2007.07.050.

[14] P. P. Korovkin, "Convergence of linear positive operators in the spaces of continuous functions" (in Russian), Doklady akademii nauk, vol. 90, pp. 961-964, 1953. 
[15] S. Mohiuddine, A. Alotaibi, and M. Mursaleen, "Statistical summability $(C, 1)$ and a Korovkin type approximation theorem", Journal of inequalities and applications, vol. 2012, no. 1, p. 172, Aug. 2012, doi: 10.1186/1029-242X-2012-172.

[16] M. A. Özarslan, O. Duman, and H. M. Srivastava, "Statistical approximation results for Kantorovich-type operators involving some special polynomials", Mathematical and computer modelling, vol. 48, no. 3-4, pp. 388-401, Aug. 2008, doi: 10.1016/j.mcm.2007.08.015.

[17] T. Pradhan, S. K. Paikray, B. B. Jena, and H. Dutta, "Statistical deferred weighted B-summability and its applications to associated approximation theorems", Journal of inequalities and applications, vol. 2018, no. 1, p. 65, Mar. 2018, doi: 10.1186/s13660-018-1650-x.

[18] H. M. Srivastava, B. B. Jena, S. K. Paikray, and U. K. Misra, "A certain class of weighted statistical convergence and associated Korovkin-type approximation theorems involving trigonometric functions", Mathematical methods in the applied sciences, vol. 41, no. 1, pp. 671-683, Jan. 2018, doi: 10.1002/mma.4636.

[19] H. M. Srivastava, B. B. Jena, S. K. Paikray, and U. K. Misra, "Generalized equistatistical convergence of the deferred Nörlund summability and its applications to associated approximation theorems", Revista de la Real Academia de ciencias exactas, físicas y naturales. Serie A. Matemáticas, vol. 112, no. 4, pp. 1487-1501, Sep. 2017, doi: 10.1007/s13398-017-0442-3.

[20] H. M. Srivastava, B. B. Jena, S. K. Paikray, and U. K. Misra, "Deferred weighted Astatistical convergence based upon the (p,q)-Lagrange polynomials and its applications to approximation theorems", Journal of applied analysis, vol. 24, no. 1, pp. 1-16, Jun. 2018, doi: 10.1515/jaa-2018-0001.

[21] H. M. Srivastava and M. Et, "Lacunary statistical convergence and strongly lacunary summable functions of order $\alpha^{\prime \prime}$, Filomat, vol. 31, no. 6, pp. 1573-1582, 2017, doi: 10.2298/fil1706573s.

[22] H. M. Srivastava, M. Mursaleen, A. M. Alotaibi, M. Nasiruzzaman, and A. A. H. AlAbied, "Some approximation results involving the q-Szász-Mirakjan-Kantorovich type operators via Dunkls generalization", Mathematical methods in the applied sciences, vol. 40, no. 15, pp. 5437-5452, Apr. 2017, doi: $10.1002 / \mathrm{mma} .4397$.

[23] H. M. Srivastava, M. Mursaleen, and A. Khan, "Generalized equi-statistical convergence of positive linear operators and associated approximation theorems", Mathematical and computer modelling, vol. 55, no. 9-10, pp. 2040-2051, May 2012, doi: 10.1016/j.mcm.2011.12.011.

[24] H. Steinhaus, "Sur la convergence ordinaire et la convergence asymptotique", Colloquium mathematicum, vol. 2, pp. 73-74, 1951.

[25] O. V. Viskov and H. M. Srivastava, "New approaches to certain identities involving differential operators", Journal of mathematical analysis and applications, vol. 186, no. 1, pp. 1-10, Aug. 1994, doi: 10.1006/jmaa.1994.1281.

[26] A. Zygmund, Trigonometric series, Cambridge: Cambridge University Press, 1979. 